\title{
Effect of 6-month community-based exercise interventions on gait and functional fitness of an older population: a quasi-experimental study
}

This article was published in the following Dove Press journal:

Clinical Interventions in Aging

\author{
Fátima Ramalho ${ }^{1,2}$ \\ Rita Santos-Rochal,2 \\ Marco Branco ${ }^{1,2}$ \\ Vera Moniz-Pereira ${ }^{2}$ \\ Helô-Isa André2 \\ António P Veloso ${ }^{2}$ \\ Filomena Carnide ${ }^{2}$ \\ 'Sport Sciences School of Rio Maior \\ (ESDRM), Polytechnic Institute \\ of Santarém, Rio Maior, Portugal; \\ ${ }^{2}$ Laboratory of Biomechanics \\ and Functional Morphology, \\ Interdisciplinary Centre for the Study \\ of Human Performance (CIPER), \\ Faculty of Human Kinetics (FMH), \\ University of Lisbon, Cruz Quebrada, \\ Portugal
}

Background: Gait ability in older adults has been associated with independent living, increased survival rates, fall prevention, and quality of life. There are inconsistent findings regarding the effects of exercise interventions in the maintenance of gait parameters.

Objectives: The aim of the study was to analyze the effects of a community-based periodized exercise intervention on the improvement of gait parameters and functional fitness in an older adult group compared with a non-periodized program.

Methods: A quasi-experimental study with follow-up was performed in a periodized exercise group $(\mathrm{N}=15)$ and in a non-periodized exercise group $(\mathrm{N}=13)$. The primary outcomes were plantar pressure gait parameters, and the secondary outcomes were physical activity, aerobic endurance, lower limb strength, agility, and balance. These variables were recorded at baseline and after 6 months of intervention.

Results: Both programs were tailored to older adults' functional fitness level and proved to be effective in reducing the age-related decline regarding functional fitness and gait parameters. Gait parameters were sensitive to both the exercise interventions.

Conclusion: These exercise protocols can be used by exercise professionals in prescribing community exercise programs, as well as by health professionals in promoting active aging.

Keywords: mobility, community exercise programs, active aging, plantar pressure analysis, ground reaction forces, gait properties

\section{Plain language summary}

Gait ability is related to mobility, which is a critical variable in maintaining independent living in older adults. Moreover, gait ability has been associated with increased survival rates, fall prevention, and quality of life of older adults. There is evidence that physical exercise interventions may prevent falls and improve the overall fitness. This raised the possibility that exercise would be effective in the maintenance of selected gait parameters. We implemented two types of exercise interventions for 6 months, in two groups of older adults to verify whether they would have a positive effect in maintaining plantar pressure gait parameters and functional fitness. Moreover, we compared these two exercise interventions to verify which would be more effective. We conclude that both the programs are proved to be effective in reducing the age-related decline regarding functional fitness and gait parameters. Effective exercise protocols can be used by exercise professionals in prescribing community exercise programs, as well as by health professionals in promoting active aging.

\section{Introduction}

Gait ability in older adults has been associated with increased survival rates, fall prevention, and quality of life. ${ }^{1}$ The changes in the structure and function of the foot are associated with aging and have considerable implications for the well-being of
Correspondence: Rita Santos-Rocha Sport Sciences School of Rio Maior (ESDRM), Polytechnic Institute of 2040-4I3, Rio Maior, Portugal

Tel +35I 966036856

Email ritasantosrocha@

esdrm.ipsantarem.pt 
the older adult. ${ }^{1}$ Gait ability is related to mobility, which in turn, is a critical variable in maintaining independence in older adults. ${ }^{2}$ Furthermore, the functional status of older adults promotes independent living, improves the quality of life, and reduces health care costs. ${ }^{1}$ Moreover, gait variability and speed can also help in identifying different categories of frailty. ${ }^{3}$

The information derived from podobarometric measures is important in gait and posture research for diagnosing lower limb problems and injury prevention. ${ }^{4}$ Several factors have been associated with high levels of plantar pressure (ie, peak pressure and pressure-time integral [PTI]) generated during gait, such as increased body weight (BW) and foot structure (arch type), ${ }^{5}$ foot pain and risk of falls, ${ }^{6}$ active diabetic foot ulcers, ${ }^{7}$ and walking strategy. ${ }^{8}$

Exercise is considered a key intervention for improving physical function in older adults. ${ }^{9}$ It is well established in the literature that exercise programs reduce the age-related decline in functional capacity and maintain muscle strength and mass among adults aged $65-85$ years. ${ }^{10}$ It is expected that $50 \%$ of reduction in the relative risk of developing functional limitations or disability was reported among those participating in a moderate-intensity physical activity (PA), ${ }^{10}$ as well as the improvement of functional fitness (FF) parameters. ${ }^{11,12}$

Recently, Wonneberger and Schmidt ${ }^{13}$ suggested that a walking-based exercise may contribute to improve step cadence and step length in multiple sclerosis patients, and Wang et $\mathrm{al}^{9}$ proved that short-term combined exercise improves gait performance in community-dwelling older adults. Different types of exercise interventions from randomized controlled trials have been shown to improve preferred gait speed. ${ }^{14}$

However, most studies were focused on clinical populations, and the heterogeneity of the interventions leads to inconsistent results on outcomes. ${ }^{15}$ In particular, there is heterogeneity regarding the ways of defining and measuring gait ability in older adults, such as a performance-based test or usual gait speed. ${ }^{16}$ Foot loading data (plantar pressure and force) can be used to analyze the effects of a treatment or an exercise intervention ${ }^{15,17}$ since plantar pressure values present a consistent pattern in the elderly, and there is no age dependence of podobarometric data. ${ }^{18,19}$ Nevertheless, the podobarometric effects of PA have rarely been investigated and reported in the literature. To the extent of our knowledge, the study developed by Monteiro et $\mathrm{al}^{20}$ is the only study that aimed to investigate the effectiveness of a 12-month exercise program on podobarometric data in postmenopausal women and has proved that women who exercised have decreased loading of maximal peak pressures and absolute impulses.
Furthermore, although periodization is an important method for training in athletes, there is still a lack of evidence of its use in untrained adults. ${ }^{21}$

Considering the importance of gait ability for the independence and quality of life of older adults, the aim of the study was to analyze the effects of a community-based periodized exercise intervention on the improvement of gait parameters and FF in an older adult group compared with a non-periodized program.

We hypothesized that a 6-month community-based periodized exercise intervention would be more effective than a non-periodized one for the maintenance or improvement of the gait and FF parameters of older participants.

\section{Methods}

\section{Design}

A quasi-experimental study was developed. The full protocol of the study is published elsewhere. ${ }^{22}$ A multicomponent community-based exercise intervention named "More Active Ageing Program" (MAAP), organized in a groupbased setting, was developed. The exercise group received a periodized intervention. The control group was committed to a non-periodized intervention. The intervention was carried out for 24 weeks, twice a week, each session spanning 50 minutes. We compared the MAAP (exercise group 1 = EG1) with other non-periodized program (NOPP) (exercise group 2 = EG2) for older adults, also described in Ramalho et al. ${ }^{22}$

The study was conducted in partnership with Sport Sciences School of Rio Maior of the Polytechnic Institute of Santarém and the research center CIPER of the Faculty of Human Kinetics of the University of Lisbon. This study received ethical approval from the Ethics Committee of the Faculty of Human Kinetics, University of Lisbon.

\section{Setting}

The intervention was implemented in six community centers, in five municipalities of West and Ribatejo regions of Portugal, allowing the recruitment of participants living in small towns and rural areas. The exercise program "MAAP" (EG1) was implemented in three of these centers. The other groups attended the exercise program "NOPP" (EG2) in the other three settings. Eligible individuals were invited to attend a formal in-person screening and then to participate in one of the community centers without any payment.

\section{Participants}

Participants were considered eligible for the study if they were aged $\geq 65$ years, community-dwelling living, understand 
Portuguese language, and agree to participate voluntarily in this study. The exclusion criteria were to have a neurologic condition (eg, dementia or Parkinson disease), a cardiovascular condition (eg, stroke), or other abnormality that would influence the ability to answer a questionnaire, inadequate proficiency in Portuguese language, to use a walking aid, presence of hip, knee, or ankle prosthesis, foot disorders, or incapacity of performing exercises in the standing position. Older male and female adults were recruited through advertisements posted within the local community.

Participants were randomly assigned to each exercise group (EG1 or EG2) taking into account the geographical proximity of their homes and intervention settings, that is, they were given the opportunity to choose between, at least, two of the available settings. The participants of both the groups were assessed at baseline and after 6 months of intervention. A maximal of 20 participants performing exercise at the same time were allowed due to practical, pedagogical, and methodologic issues related to the training.

Of a total of 40 participants recruited for the intervention, 20 were engaged in the EG1, and other 20 older adults were engaged in the EG2. Since they missed (or "because they have missed") the second assessment, five subjects were excluded from the analysis in EG1 (12.5\% loss of follow-up) and seven in EG2 (17.5\% loss of follow-up).

Fifteen participants of the EG1 (mean age of $68.0 \pm 5.9$ years at baseline; $66.0 \pm 16.4 \mathrm{~kg}$ of body mass; $85 \%$ females) and 13 participants of the EG2 (mean age of $67.8 \pm 5.2$ years at baseline; $72.1 \pm 10.3 \mathrm{~kg}$ of body mass; $85 \%$ females) were assessed. Figure 1 depicts the recruitment, randomization, and assessment processes.

The project manager explained the objectives of the study and answered all questions before asking the participant to consent the participation in the study. A written informed consent was obtained from all participants at the beginning of the intervention, before the allocation to the groups. All participants were instructed to maintain their normal lifestyle through the intervention.

\section{Exercise intervention}

All exercise sessions included in both the exercise programs were delivered by different graduated exercise specialists, blind to the intervention. Participants were instructed to wear comfortable sportswear and sports shoes during both the interventions.

EG1 was engaged in an exercise program MAAP, which was controlled, periodized, and organized, according to the structure presented in Ramalho et al. ${ }^{22}$ The rational is also

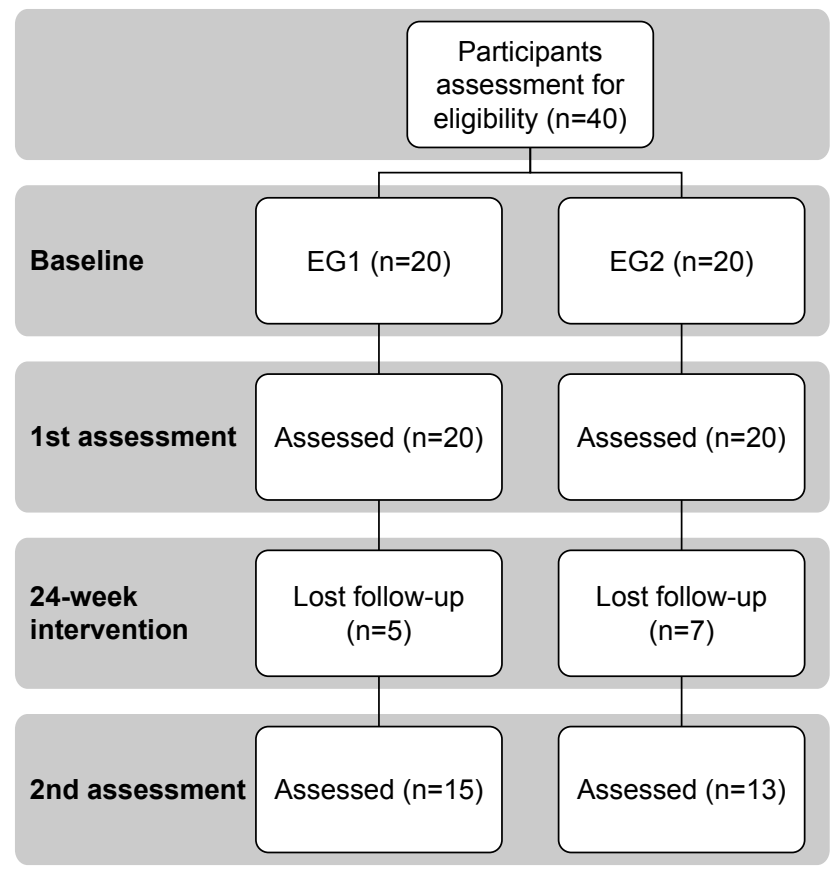

Figure I Flow diagram of the recruitment, randomization, and assessment processes. Abbreviation: EG, exercise group.

explained in the study protocol. ${ }^{22}$ The MAAP was designed to focus specifically on the postural stability, balance, and strength in lower limbs, combined with aerobic exercise, at a level of intensity in accordance with the participants' functional capacities. The components of posture stability, balance, and strength training were based on group exercise and circuit training. The aerobic component was based on walking and mobility group exercise. During the first period of 12 weeks, more emphasis was given to the development of postural control and balance, and the second period of 12 weeks was more focused on strength and muscle resistance improvement in lower limbs. The exercise specialists responsible for NOPP (EG2) followed the same planning on all subgroup classes, in accordance with the recommendations provided by the American College of Sports Medicine (ACSM) ${ }^{23}$ and Rose. ${ }^{24}$ The NOPP program was designed to focus on balance, strength, and aerobic exercise, without any specific periodization. A more detailed description of this exercise program is also described in Ramalho et al. ${ }^{22}$

The participation of all subjects was controlled through attendance forms and session goal checklists. Participants with an attendance rate $<75 \%$ of the sessions in each of the programs' mesocycles would be excluded from the analysis. However, all participants attended at least $95 \%$ of the sessions. The loss of follow-up refers to participants who missed one of the assessments, and for that reason, they were excluded from the analysis (Figure 1). 
The independent variables of this study were the features (type, duration, frequency, intensity, and progression/ periodization) of the exercise programs.

\section{Testing procedures}

All measurements were applied using standardized protocols and trained researchers.

\section{Gait assessment}

Gait parameters were obtained using a Novel emed-x system platform (Novel, Münich, Germany) with a high sensor resolution mode with a sensor resolution of 4 sensors $/ \mathrm{cm}^{2}$ and a frame rate of $100 \mathrm{~Hz}$. Gait parameters were recorded as participants walked barefoot across a $10 \mathrm{~m}$ walkway placed on the floor, at self-determined velocity. The participants performed few trials before data collection, in order to be familiarized with the task. Five stances from each foot from each participant were collected and analyzed. Portable equipment was used to collect data in the same setting where the exercise interventions and the other assessments took place.

The primary outcome variables were the following gait parameters: 1) applied local force-maximum vertical ground reaction force (max force); 2) maximum applied local pressure (peak pressure); 3) maximum mean applied pressure (max mean pressure); 4) force-time integral (FTI) in an anatomical area; 5) PTI. For subsequent analysis of force and plantar pressure, each foot (right and left) was divided into six anatomical areas: "hindfoot," "midfoot," "medial forefoot," "hallux," "toes," and "lateral forefoot" (metatarsal heads), plus "total foot." The total foot and the six foot areas selected for analysis are depicted in Figure 2.

The Novel software was used for data analysis. Force data were normalized to the BW. This process allows better comparisons between participants since it eliminates differences in BW as a potential confounder during statistical analysis. Since a previous comparison showed no significant differences between right and left foot, the mean values of both feet were used in the subsequent statistical analysis.

\section{FF assessment}

FF tests included in the "Senior Fitness Tests" (SFT) battery ${ }^{25}$ were administered by trained examiners after engaging in a training workshop, which previously demonstrated a high inter-observer agreement for all FF tests. ${ }^{26}$ The tests " $30 \mathrm{sec}$ chair stand," "8 ft up\&go," and "2-min walking" were used to assess lower limb strength, agility, and aerobic endurance, respectively. Both dynamic and static balance were evaluated through two tests each (FAB 1-4), included in the "Fullerton

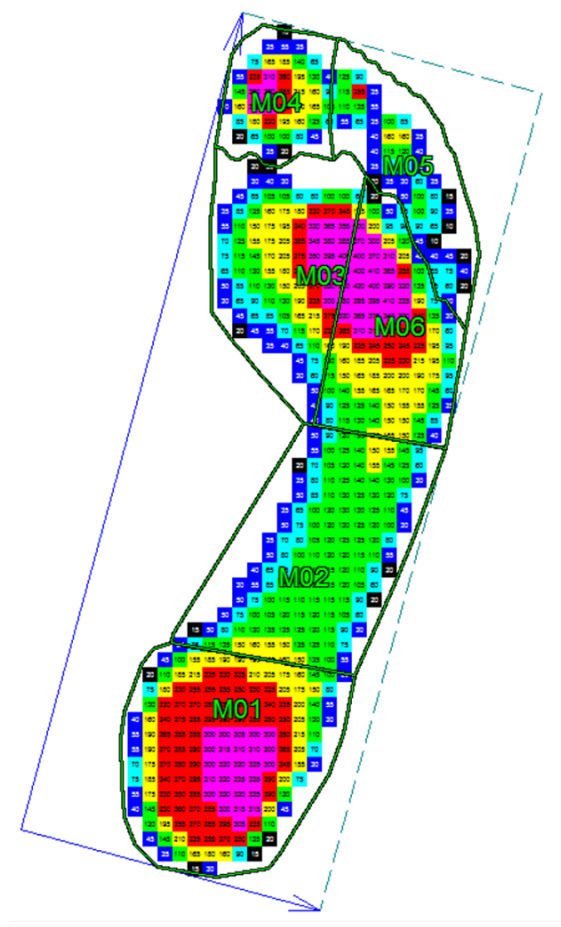

Figure 2 The six foot regions in analysis: MOI - hindfoot (heel); M02 - midfoot; M03 - medial forefoot; M04 - hallux; M05 - toes; M06 - lateral forefoot.

Advanced Balance Scale." ${ }^{24}$ These FF parameters were the secondary variables of the study. All participants followed a familiarization session of 15 minutes ( 2 minutes for each test) 1 week before the baseline assessments.

\section{Demographics, health, and physical activity assessment}

The characteristics of the participants included in the study were demographics, self-perception of health and falls prevalence, medication intake, PA levels, body mass, height, and body mass index (BMI).

Self-perception of health status and falls prevalence defined as the experience of any falls during the period before assessments: previous 12 months at baseline and during the follow-up period - were assessed by a questionnaire designed and validated for Portuguese language and culture. ${ }^{27}$

PA profiles were also evaluated by a short version of "Yale Physical Activity Survey" (YPAS), validated for the Portuguese population, ${ }^{28}$ which defines six distinct PA dimensions: 1) vigorous activity, 2) leisurely walking, 3) moving, 4) standing, and 5) sitting, and a PA total score, considering a typical week. Both questionnaires were administered by face-to-face interviews involving the same trained examiners. The procedures are described elsewhere. ${ }^{26}$

Height $(\mathrm{m})$ and body mass $(\mathrm{kg})$ were assessed using a stadiometer and calibrated scale SECA (model 220; SECA, Hamburg, Germany). BMI was calculated as $\mathrm{kg} / \mathrm{m}^{2}$. 


\section{Statistical analysis}

Statistical analysis was performed using the Statistical Package for Social Sciences (SPSS version 23.0) to obtain descriptive statistics (mean and standard deviation) and to evaluate the effectiveness of the MAAP on the improvement of gait and FF parameters in the EG1, compared to the NOPP administered to the EG2. Data normality distribution was checked with the Shapiro-Wilk test. Paired $t$-tests (and the non-parametric Wilcoxon tests) were used to compare data at baseline and after 6 months of intervention. Independent sample $t$-tests (and the non-parametric Mann-Whitney $U$ tests) were performed to compare the outcomes between the groups (EG1 versus EG2). Statistical significance was set at $p<0.05$.

\section{Results}

The characteristics of the participants, as well as the results of FF parameters (secondary variables) in both the groups, at baseline and after the exercise intervention, are summarized in Table 1.

Regarding both the exercise interventions, none of the participants reported any injury or complications in their feet during the intervention period and showed no signs of excessive fatigue or discomfort during the assessments.
There were no differences between groups, at baseline and after the intervention, regarding self-perception of health ( $3.5 \pm 0.4$ score units), number of intake medications (2.5 \pm 1.3$)$, and falls prevalence in the last year $(0.2 \pm 0.1)$. There were no differences between groups regarding the participants' BW, BMI, and PA level, neither at baseline nor after the intervention, except for "moving score" which increased after the intervention.

Regarding the FF variables, there are no differences between groups at baseline. The results point to the maintenance of most FF parameters after the 24-week exercise intervention, except for the " $30 \mathrm{sec}$ chair-stand" test that increased in EG2 after intervention and in comparison with EG1.

The results of gait parameters (primary outcomes) in both the groups at baseline and after the exercise intervention are summarized in Tables $2-5$.

At baseline, there were no significant differences between groups regarding the analyzed variables (Table 2 ). The significant changes between baseline and after the intervention in both the groups are depicted in Figures 3-7.

Regarding the potential effects of the MAAP, the following parameters showed a significant decrease after the intervention: maximal force in the hallux; maximal mean pressure in all foot, toes, and lateral forefoot; peak pressure in all foot

Table I Characteristics of the participants and functional fitness results in both the exercise groups, at baseline and after the 24-week exercise intervention

\begin{tabular}{|c|c|c|c|c|c|}
\hline \multirow[t]{4}{*}{ Factors } & \multirow{2}{*}{\multicolumn{2}{|c|}{$\begin{array}{l}\text { Exercise group I (EGI) } \\
N=15\end{array}$}} & \multirow{2}{*}{\multicolumn{2}{|c|}{$\begin{array}{l}\text { Exercise group } 2 \text { (EG2) } \\
N=13\end{array}$}} & \multirow[t]{4}{*}{$\mathbf{p}<0.05$} \\
\hline & & & & & \\
\hline & \multirow{2}{*}{$\begin{array}{l}\text { Baseline } \\
\text { Mean } \pm \text { SD }\end{array}$} & \multirow{2}{*}{$\begin{array}{l}\text { After } \\
\text { intervention } \\
\text { Mean } \pm \text { SD }\end{array}$} & \multirow{2}{*}{$\begin{array}{l}\text { Baseline } \\
\text { Mean } \pm \text { SD }\end{array}$} & \multirow{2}{*}{$\begin{array}{l}\text { After } \\
\text { intervention } \\
\text { Mean } \pm \text { SD }\end{array}$} & \\
\hline & & & & & \\
\hline Body weight $(\mathrm{N})$ & $653.01 \pm 81.23$ & $647.78 \pm 80.04$ & $676.3 \pm 88.8$ & $674.0 \pm 82.8$ & \\
\hline Body mass index $\left(\mathrm{kg} / \mathrm{m}^{2}\right)$ & $27.2 \pm 3.5$ & $26.4 \pm 3.3$ & $28.0 \pm 2.5$ & $27.6 \pm 2.6$ & \\
\hline Vigorous walking (score units) & $5.3 \pm 12.0$ & $9.7 \pm 12.6$ & $15.4 \pm 16.2$ & $9.6 \pm 9.6$ & \\
\hline Walking (score units) & $9.3 \pm 5.2$ & II. $2 \pm 7.7$ & $13.0 \pm 12.8$ & $18.6 \pm 13.7$ & a \\
\hline Moving (score units) & $10.5 \pm 3.2$ & $11.2 \pm 2.4$ & $12.8 \pm 1.4$ & $13.1 \pm 1.5$ & $*$ \\
\hline Standing (score units) & $7.9 \pm 1.8$ & $8.4 \pm 1.9$ & $9.0 \pm 1.0$ & $9.3 \pm 1.0$ & \\
\hline Sitting (score units) & $2.3 \pm 0.8$ & $2.3 \pm 0.8$ & $2.3 \pm 0.5$ & $2.1 \pm 0.5$ & \\
\hline YPAS total (score units) & $51.3 \pm 12.6$ & $42.7 \pm 16.5$ & $52.4 \pm 17.8$ & $52.6 \pm 15.4$ & \\
\hline $\begin{array}{l}30 \mathrm{sec} \text { chair-stand } \\
\text { (number of repetitions) }\end{array}$ & $15.3 \pm 3.7$ & $16.4 \pm 2.8$ & $16.3 \pm 3.1$ & $21.2 \pm 5.8$ & $\#, \S$ \\
\hline $8 \mathrm{ft}$ up\&go (score units) & $5.5 \pm 1.2$ & $5.0 \pm 0.7$ & $4.8 \pm 0.8$ & $5.1 \pm 0.8$ & \\
\hline 2-min walking (score units) & $105.9 \pm 21.4$ & $111.7 \pm 17.5$ & $110.8 \pm 21.5$ & $111.8 \pm 25.1$ & \\
\hline FABI (score units) & $4.00 \pm 0.0$ & $4.0 \pm 0.0$ & $4.0 \pm 0.0$ & $4.0 \pm 0.0$ & \\
\hline FAB2 (score units) & $3.3 \pm 1.0$ & $3.7 \pm 0.5$ & $3.3 \pm 1.0$ & $3.6 \pm 0.7$ & \\
\hline FAB3 (score units) & $3.2 \pm 1.2$ & $3.3 \pm 1.2$ & $3.3 \pm 1.0$ & $3.3 \pm 0.8$ & \\
\hline FAB4 (score units) & $3.7 \pm 1.0$ & $3.7 \pm 0.6$ & $3.9 \pm 0.3$ & $3.9 \pm 0.3$ & \\
\hline
\end{tabular}

Notes: ${ }^{\sharp}$ Significant differences between EGI and EG2 after intervention; "significant differences between baseline and after intervention in EGI; ${ }^{\S}$ significant differences between baseline and after intervention in EG2. "30 sec chair-stand" (CS), lower limbs strength test; "8 foot up\&go" (UP), agility test; "2-min walking", aerobic endurance test; $F A B I$ and $F A B 2$, dynamic balance tests; FAB3 and FAB4, static balance tests.

Abbreviations: EG, exercise group; YPAS, Yale Physical Activity Survey. 
Table 2 Comparisons of the results of gait parameters between exercise group I and exercise group 2, at baseline

\begin{tabular}{|c|c|c|c|}
\hline \multirow[t]{2}{*}{$\begin{array}{l}\text { Gait } \\
\text { parameters }\end{array}$} & \multirow{2}{*}{$\begin{array}{l}\text { Exercise } \\
\text { group I } \\
(\mathrm{N}=15) \\
\text { Mean } \pm \text { SD }\end{array}$} & \multirow{2}{*}{$\begin{array}{l}\text { Exercise } \\
\text { group } 2 \\
(\mathrm{~N}=13) \\
\text { Mean } \pm \mathrm{SD}\end{array}$} & \multirow[t]{2}{*}{$\begin{array}{l}\text { Significance } \\
\text { (two-tailed) }\end{array}$} \\
\hline & & & \\
\hline All_FTI & $56.0 \pm 3.8$ & $57.7 \pm 9.2$ & 0.905 \\
\hline All_PeakF & $115.7 \pm 5.8$ & $116.4 \pm 9.4$ & 0.548 \\
\hline All_MeanP & $222.7 \pm 56.6$ & $203.6 \pm 36.1$ & 0.456 \\
\hline All_PeakP & $641.2 \pm 194.1$ & $534.8 \pm 69.4$ & 0.183 \\
\hline All_PTI & $226.3 \pm 58.1$ & $208.5 \pm 41.6$ & 0.381 \\
\hline MOI_FTI & $16.5 \pm 2.6$ & $15.2 \pm 2.6$ & 0.215 \\
\hline MOI_PeakF & $71.1 \pm 9.1$ & $67.5 \pm 10.6$ & 0.342 \\
\hline MOI_MeanP & $100.9 \pm 28.5$ & $86.8 \pm 13.9$ & 0.236 \\
\hline MOI_PeakP & $327.2 \pm 111.6$ & $293.1 \pm 59.8$ & 0.614 \\
\hline MOI_PTI & $74.8 \pm 21.4$ & $66.1 \pm 10.6$ & 0.456 \\
\hline M02_FTI & $5.5 \pm 3.4$ & $5.7 \pm 2.9$ & 0.846 \\
\hline M02_PeakF & $19.9 \pm 9.4$ & $21.7 \pm 9.0$ & 0.624 \\
\hline M02_MeanP & $47.2 \pm 12.6$ & $49.8 \pm 14.3$ & 0.627 \\
\hline M02_PeakP & $|35.1 \pm 3| .5$ & $146.9 \pm 27.8$ & 0.319 \\
\hline M02_PTI & $39.5 \pm 12.6$ & $42.9 \pm 16.1$ & 0.537 \\
\hline M03_FTI & $29.3 \pm 4.1$ & $31.0 \pm 4.7$ & 0.335 \\
\hline M03_PeakF & $95.8 \pm 8.3$ & $91.3 \pm 5.4$ & 0.114 \\
\hline M03_MeanP & $217.4 \pm 60.1$ & $186.5 \pm 34.9$ & 0.256 \\
\hline M03_PeakP & $615.5 \pm 2 \mid 1.4$ & $487.6 \pm 72.4$ & 0.152 \\
\hline M03_PTI & $169.4 \pm 52.5$ & $147.4 \pm 30.6$ & 0.427 \\
\hline M04_FTI & $3.2 \pm 1.357$ & $3.9 \pm 1.3$ & 0.148 \\
\hline M04_PeakF & $16.4 \pm 6.7$ & $16.8 \pm 4.8$ & 0.844 \\
\hline M04_MeanP & $91.3 \pm 40.7$ & $116.2 \pm 39.8$ & 0.123 \\
\hline M04_PeakP & $348.1 \pm 144.0$ & $366.4 \pm 89.8$ & 0.706 \\
\hline M04_PTI & $69.7 \pm 32.2$ & $94.0 \pm 51.2$ & 0.139 \\
\hline M05_FTI & $1.6 \pm 0.6$ & $2.0 \pm 1.3$ & 0.792 \\
\hline M05_PeakF & $7.7 \pm 2.9$ & $8.4 \pm 4.1$ & 0.599 \\
\hline M05_MeanP & $56.4 \pm 19.1$ & $62.8 \pm 35.0$ & 0.648 \\
\hline M05_PeakP & $182.8 \pm 56.6$ & $193.5 \pm 97.2$ & 0.724 \\
\hline M05_PTI & $42.4 \pm 13.9$ & $52.0 \pm 36.9$ & 0.581 \\
\hline M06_FTI & $14.0 \pm 3.1$ & $15.3 \pm 3.6$ & 0.352 \\
\hline M06_PeakF & $44.6 \pm 9.6$ & $44.9 \pm 7.0$ & 0.932 \\
\hline M06_MeanP & $|87.1 \pm 5| .3$ & $158.5 \pm 24.4$ & 0.300 \\
\hline M06_PeakP & $493.1 \pm 152.8$ & $404 . I \pm 43.6$ & 0.126 \\
\hline M06_PTI & $140.6 \pm 43.6$ & $122.5 \pm 18.9$ & 0.829 \\
\hline
\end{tabular}

Abbreviations: $\mathrm{FTI}$, force-time integral in an anatomical area; PTI, pressure-time integral; PeakF, applied local force - maximum vertical ground reaction force; PeakP, maximum applied local pressure; MeanP, maximum mean applied pressure; M0I, hindfoot; M02, midfoot; M03, medial forefoot; M04, hallux; M05, toes; M06, lateral forefoot.

and medial forefoot; and PTI in toes and lateral forefoot. There was no change in the remaining assessed parameters.

For NOPP, the following parameters showed a significant decrease after the intervention: FTI in all foot, midfoot, and medial forefoot; maximal mean pressure in toes and lateral forefoot; peak pressure in the medial forefoot; and PTI in all foot, toes, and lateral forefoot. There was no change in the remaining assessed parameters.
Table 3 Comparisons of results of gait parameters between baseline and after exercise intervention in exercise group I

\begin{tabular}{|c|c|c|c|}
\hline \multirow[t]{2}{*}{$\begin{array}{l}\text { Gait } \\
\text { parameters }\end{array}$} & \multirow{2}{*}{$\begin{array}{l}\text { Exercise } \\
\text { group I } \\
(\mathrm{N}=15) \\
\text { Mean } \pm \text { SD }\end{array}$} & \multirow{2}{*}{$\begin{array}{l}\text { Exercise } \\
\text { group } 2 \\
(\mathrm{~N}=\mid 3) \\
\text { Mean } \pm \mathrm{SD}\end{array}$} & \multirow[t]{2}{*}{$\begin{array}{l}\text { Significance } \\
\text { (two-tailed) }\end{array}$} \\
\hline & & & \\
\hline All_FTI & $56.0 \pm 3.8$ & $55.2 \pm 5.2$ & 0.650 \\
\hline All_PeakF & $115.7 \pm 5.8$ & $114.0 \pm 6.0$ & 0.394 \\
\hline All_MeanP & $222.7 \pm 56.6$ & $211.1 \pm 47.0$ & $0.04 I$ \\
\hline All_PeakP & $641.2 \pm 194.1$ & $606.5 \pm 164.7$ & 0.027 \\
\hline All_PTI & $226.3 \pm 58.1$ & $216.1 \pm 46.7$ & 0.256 \\
\hline MOI_FTI & $16.5 \pm 2.6$ & $16.5 \pm 2.3$ & 0.865 \\
\hline MOI_PeakF & $71.1 \pm 9.1$ & $72.5 \pm 9.6$ & 0.532 \\
\hline MOI_MeanP & $100.9 \pm 28.5$ & $102.9 \pm 26.2$ & 0.439 \\
\hline MOI_PeakP & $327.2 \pm 111.6$ & $332.5 \pm 86.7$ & 0.363 \\
\hline MOI_PTI & $74.8 \pm 21.4$ & $75.6 \pm 16.4$ & 0.532 \\
\hline M02_FTI & $5.4 \pm 3.4$ & $5.2 \pm 3.5$ & 0.349 \\
\hline M02_PeakF & $19.9 \pm 9.4$ & $18.9 \pm 9.1$ & 0.140 \\
\hline M02_MeanP & $47.2 \pm 12.6$ & $47.6 \pm 18.2$ & 0.477 \\
\hline M02_PeakP & $|35| \pm 3 \mid .5$. & $139.7 \pm 44.0$ & 0.315 \\
\hline M02_PTI & $39.5 \pm 12.6$ & $40.2 \pm 17.6$ & 0.609 \\
\hline M03_FTI & $29.3 \pm 4.1$ & $29.1 \pm 4.9$ & 0.776 \\
\hline M03_PeakF & $95.8 \pm 8.3$ & $94.8 \pm 8.2$ & 0.691 \\
\hline M03_MeanP & $217.4 \pm 60.1$ & $206.9 \pm 48.7$ & 0.086 \\
\hline M03_PeakP & $615.5 \pm 211.4$ & $58 I .7 \pm I 77.5$ & 0.019 \\
\hline M03_PTI & $169.4 \pm 52.5$ & $159.1 \pm 46.9$ & 0.075 \\
\hline M04_FTI & $3.2 \pm 1.4$ & $2.9 \pm 1.1$ & $0.28 \mathrm{I}$ \\
\hline M04_PeakF & $16.4 \pm 6.7$ & $15.3 \pm 6.7$ & 0.017 \\
\hline M04_MeanP & $91.3 \pm 40.7$ & $86.8 \pm 36.4$ & 0.426 \\
\hline M04_PeakP & $348.1 \pm 144.0$ & $329.3 \pm 143.1$ & 0.140 \\
\hline M04_PTI & $69.7 \pm 32.2$ & $65.8 \pm 28.7$ & 0.406 \\
\hline M05_FTI & $1.6 \pm 0.6$ & $1.4 \pm 0.7$ & 0.129 \\
\hline M05_PeakF & $7.7 \pm 2.9$ & $7.4 \pm 3.8$ & 0.820 \\
\hline M05_MeanP & $56.4 \pm 19.1$ & $49.1 \pm 21.0$ & 0.036 \\
\hline M05_PeakP & $182.8 \pm 56.6$ & $169.2 \pm 66.2$ & 0.201 \\
\hline M05_PTI & $42.4 \pm I 3.8$ & $37.4 \pm I 4.4$ & 0.047 \\
\hline M06_FTI & $14.0 \pm 3.1$ & $13.8 \pm 3.2$ & 0.532 \\
\hline M06_PeakF & $44.6 \pm 9.6$ & $43.9 \pm 9.2$ & 0.609 \\
\hline M06_MeanP & $|87.1 \pm 5| .3$ & $|76.4 \pm 4| .5$ & 0.027 \\
\hline M06_PeakP & $493.1 \pm 152.8$ & $469.0 \pm 131.8$ & 0.062 \\
\hline M06_PTI & $140.6 \pm 43.6$ & $132.0 \pm 40.3$ & 0.028 \\
\hline
\end{tabular}

Note: Significant differences in bold case.

Abbreviations: $\mathrm{FTI}$, force-time integral in an anatomical area; PTI, pressure-time integral; PeakF, applied local force - maximum vertical ground reaction force; PeakP, maximum applied local pressure; MeanP, maximum mean applied pressure; MOI, hindfoot; M02, midfoot; M03, medial forefoot; M04, hallux; M05, toes; M06, lateral forefoot.

After the intervention, significant differences were observed between groups in the following parameters: EG1 showed higher values than EG2 in FTI and PTI in the hindfoot region (Table 5).

\section{Discussion}

The aim of the study was to analyze the effects of a 6-month community-based periodized exercise intervention on the 
Table 4 Comparisons of results of gait parameters between baseline and after exercise intervention in exercise group 2

\begin{tabular}{|c|c|c|c|}
\hline \multirow[t]{2}{*}{$\begin{array}{l}\text { Gait } \\
\text { parameters }\end{array}$} & \multirow{2}{*}{$\begin{array}{l}\text { Exercise } \\
\text { group I } \\
(\mathrm{N}=15) \\
\text { Mean } \pm \text { SD }\end{array}$} & \multirow{2}{*}{$\begin{array}{l}\text { Exercise } \\
\text { group } 2 \\
(\mathbf{N}=13) \\
\text { Mean } \pm \mathrm{SD}\end{array}$} & \multirow[t]{2}{*}{$\begin{array}{l}\text { Significance } \\
\text { (two-tailed) }\end{array}$} \\
\hline & & & \\
\hline All_FTI & $57.7 \pm 9.2$ & $52.0 \pm 5.7$ & 0.015 \\
\hline All_PeakF & $116.4 \pm 9.4$ & $116.4 \pm 9.9$ & 0.937 \\
\hline All_MeanP & $203.6 \pm 36.1$ & $195.0 \pm 45.5$ & 0.209 \\
\hline All_PeakP & $534.8 \pm 69.4$ & $530.3 \pm 110.8$ & 0.814 \\
\hline All_PTI & $208.5 \pm 41.6$ & $187.9 \pm 35.3$ & 0.034 \\
\hline MOI_FTI & $15.2 \pm 2.6$ & $14.6 \pm 2.0$ & 0.480 \\
\hline MOI_PeakF & $67.5 \pm 10.3$ & $72.2 \pm 7.7$ & 0.209 \\
\hline MOI_MeanP & $86.8 \pm 13.9$ & $91.0 \pm 13.7$ & 0.439 \\
\hline MOI_PeakP & $293.1 \pm 59.8$ & $306.7 \pm 48.3$ & 0.583 \\
\hline MOI_PTI & $66.1 \pm 10.6$ & $63.8 \pm 11.7$ & 0.583 \\
\hline M02_FTI & $5.7 \pm 2.9$ & $4.7 \pm 2.2$ & 0.019 \\
\hline M02_PeakF & $21.7 \pm 9.0$ & $20.6 \pm 9.0$ & 0.308 \\
\hline M02_MeanP & $49.8 \pm 14.3$ & $46.0 \pm 12.9$ & 0.136 \\
\hline M02_PeakP & $146.9 \pm 27.7$ & $|44.3 \pm 3| .2$ & 0.784 \\
\hline M02_PTI & $42.9 \pm 16.0$ & $36.0 \pm 10.6$ & 0.019 \\
\hline M03_FTI & $31.0 \pm 4.7$ & $27.4 \pm 4.1$ & 0.019 \\
\hline M03_PeakF & $91.3 \pm 5.4$ & $90.0 \pm 5.7$ & 0.638 \\
\hline M03_MeanP & $186.5 \pm 34.9$ & I $79.3 \pm 39.7$ & 0.086 \\
\hline M03_PeakP & $487.6 \pm 72.4$ & $467.7 \pm 102.6$ & 0.019 \\
\hline M03_PTI & $147.4 \pm 30.6$ & $\mid 28.7 \pm 34.4$ & 0.075 \\
\hline M04_FTI & $3.9 \pm 1.3$ & $3.6 \pm 1.1$ & 0.388 \\
\hline M04_PeakF & $16.8 \pm 4.8$ & $17.7 \pm 5.5$ & 0.388 \\
\hline M04_MeanP & $116.2 \pm 39.8$ & $113.6 \pm 42.7$ & 0.695 \\
\hline M04_PeakP & $366.4 \pm 89.8$ & $381.1 \pm 108.2$ & 0.530 \\
\hline M04_PTI & $94.0 \pm 51.2$ & $80.3 \pm 30.3$ & 0.406 \\
\hline M05_FTI & $2.0 \pm 1.3$ & $1.7 \pm 1.0$ & 0.129 \\
\hline M05_PeakF & $8.4 \pm 4.1$ & $8.4 \pm 3.9$ & 0.875 \\
\hline M05_MeanP & $62.8 \pm 35.0$ & $62.4 \pm 29.6$ & 0.036 \\
\hline M05_PeakP & $193.4 \pm 97.2$ & $208.8 \pm 91.1$ & 0.065 \\
\hline M05_PTI & $52.0 \pm 36.9$ & $44.6 \pm 20.5$ & 0.209 \\
\hline M06_FTI & $15.2 \pm 3.6$ & $12.9 \pm 2.3$ & 0.532 \\
\hline M06_PeakF & $44.9 \pm 7.0$ & $42.9 \pm 5.6$ & 0.583 \\
\hline M06_MeanP & I $58.5 \pm 24.4$ & $153.2 \pm 19.9$ & 0.027 \\
\hline M06_PeakP & $404.1 \pm 43.6$ & $381.4 \pm 57.3$ & 0.062 \\
\hline M06_PTI & $122.5 \pm \mid 8.9$ & $106.3 \pm 17.6$ & 0.028 \\
\hline
\end{tabular}

Note: Significant differences in bold case.

Abbreviations: FTI, force-time integral in an anatomical area; PTI, pressure-time integral; PeakF, applied local force - maximum vertical ground reaction force; PeakP, maximum applied local pressure; MeanP, maximum mean applied pressure; MOI, hindfoot; M02, midfoot; M03, medial forefoot; M04, hallux; M05, toes; M06, lateral forefoot.

improvement of gait parameters and $\mathrm{FF}$ in an older adult group compared with a non-periodized program. We hypothesized that a periodized community program, in terms of its specific features (ie, type, intensity, frequency, duration, and progression), would be more effective in the improvement of gait and FF parameters within the older population, than a non-periodized exercise program.

The EG2 program increased significantly the strength in lower limbs and showed higher plantar pressure values
Table 5 Comparisons of results of gait parameters results between exercise group $I$ and exercise group 2, after exercise intervention

\begin{tabular}{|c|c|c|c|}
\hline \multirow[t]{2}{*}{$\begin{array}{l}\text { Gait } \\
\text { parameters }\end{array}$} & \multirow{2}{*}{$\begin{array}{l}\text { Exercise } \\
\text { group I } \\
(\mathrm{N}=15) \\
\text { Mean } \pm \text { SD }\end{array}$} & \multirow{2}{*}{$\begin{array}{l}\text { Exercise } \\
\text { group } 2 \\
(\mathrm{~N}=13) \\
\text { Mean } \pm \mathrm{SD}\end{array}$} & \multirow[t]{2}{*}{$\begin{array}{l}\text { Significance } \\
\text { (two-tailed) }\end{array}$} \\
\hline & & & \\
\hline All_FTI & $55.2 \pm 5.2$ & $52.0 \pm 5.7$ & 0.144 \\
\hline All_PeakF & $114.0 \pm 6.0$ & $116.5 \pm 9.9$ & 0.432 \\
\hline All_MeanP & $211.1 \pm 47.0$ & $195.0 \pm 45.5$ & 0.379 \\
\hline All_PeakP & $606.5 \pm 164.7$ & $530.3 \pm 110.8$ & 0.182 \\
\hline All_PTI & $216.1 \pm 46.7$ & $187.9 \pm 35.3$ & 0.096 \\
\hline MOI_FTI & $16.5 \pm 2.3$ & $14.6 \pm 2.0$ & 0.030 \\
\hline MOI_PeakF & $72.5 \pm 9.6$ & $72.2 \pm 7.7$ & 0.936 \\
\hline MOI_MeanP & $102.9 \pm 26.2$ & $91.0 \pm 13.7$ & 0.272 \\
\hline MOI_PeakP & $332.5 \pm 86.7$ & $306.7 \pm 48.3$ & 0.365 \\
\hline MOI_PTI & $75.6 \pm 16.4$ & $63.8 \pm I 1.7$ & 0.046 \\
\hline M02_FTI & $5.2 \pm 3.6$ & $4.7 \pm 2.2$ & 0.654 \\
\hline M02_PeakF & $18.9 \pm 9.1$ & $20.7 \pm 9.0$ & 0.627 \\
\hline M02_MeanP & $47.6 \pm 18.2$ & $46.0 \pm 12.9$ & 0.525 \\
\hline M02_PeakP & $139.7 \pm 44.0$ & $|44.3 \pm 3| .2$ & 0.760 \\
\hline M02_PTI & $40.2 \pm 17.6$ & $36.0 \pm 10.6$ & 0.922 \\
\hline M03_FTI & $29.1 \pm 4.9$ & $27.4 \pm 4.1$ & 0.361 \\
\hline M03_PeakF & $94.8 \pm 8.2$ & $90.0 \pm 5.7$ & 0.098 \\
\hline M03_MeanP & $206.9 \pm 48.7$ & $179.3 \pm 39.7$ & 0.097 \\
\hline M03_PeakP & $581.7 \pm 177.6$ & $467.7 \pm 102.6$ & 0.097 \\
\hline M03_PTI & $159.1 \pm 46.9$ & $128.7 \pm 34.4$ & 0.079 \\
\hline M04_FTI & $2.9 \pm 1.1$ & $3.6 \pm 1.1$ & 0.145 \\
\hline M04_PeakF & $15.3 \pm 6.7$ & $17.7 \pm 5.5$ & 0.337 \\
\hline M04_MeanP & $86.8 \pm 36.4$ & $113.7 \pm 42.7$ & 0.097 \\
\hline M04_PeakP & $329.3 \pm \mid 43.1$ & $381.1 \pm 108.2$ & 0.310 \\
\hline M04_PTI & $65.8 \pm 28.7$ & $80.3 \pm 30.3$ & 0.262 \\
\hline M05_FTI & $1.5 \pm 0.7$ & $1.7 \pm 1.0$ & 0.407 \\
\hline M05_PeakF & $7.4 \pm 3.8$ & $8.4 \pm 3.9$ & 0.487 \\
\hline M05_MeanP & $49.1 \pm 21.0$ & $62.4 \pm 29.6$ & 0.272 \\
\hline M05_PeakP & $169.2 \pm 66.3$ & 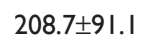 & 0.203 \\
\hline M05_PTI & $37.4 \pm 14.4$ & $44.6 \pm 20.5$ & 0.289 \\
\hline M06_FTI & $13.8 \pm 3.2$ & $12.9 \pm 2.3$ & 0.444 \\
\hline M06_PeakF & $43.9 \pm 9.2$ & $43.0 \pm 5.6$ & 0.758 \\
\hline M06_MeanP & $|76.4 \pm 4| .5$ & $153.2 \pm 19.9$ & 0.172 \\
\hline M06_PeakP & $469.0 \pm|3| .8$ & $38 I .4 \pm 57.3$ & 0.097 \\
\hline M06_PTI & $132.0 \pm 40.3$ & $106.3 \pm 17.6$ & 0.118 \\
\hline
\end{tabular}

Note: Significant differences in bold case.

Abbreviations: FTI, force-time integral in an anatomical area; PTI, pressure-time integral; PeakF, applied local force - maximum vertical ground reaction force; PeakP, maximum applied local pressure; MeanP, maximum mean applied pressure; MOI, hindfoot; M02, midfoot; M03, medial forefoot; M04, hallux; M05, toes; M06, lateral forefoot.

(FTI and PTI in the hindfoot region). The MAAP was designed to focus specifically on the postural stability, balance, and strength in lower limbs, combined with aerobic exercise, which could justify the improvement in gait stability. On the other hand, the increased effectiveness related to EG2 should be analyzed with caution, because both the groups were involved in exercise programs with the same type of exercises, differing only on the progression of the 


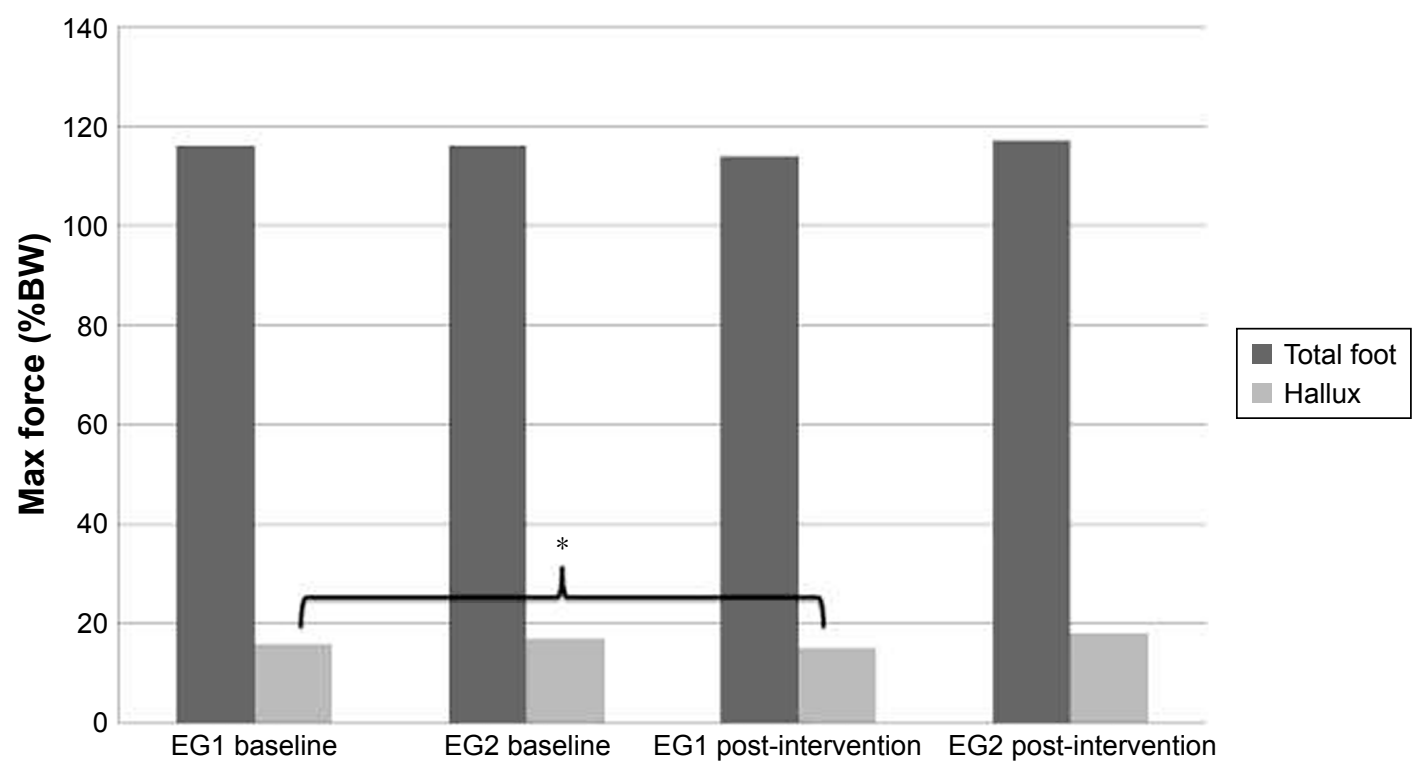

Figure 3 Significant changes between baseline and after intervention, in both the groups, regarding maximal force $\left({ }^{*} p<0.05\right)$.

Abbreviations: EGI, exercise group I; EG2, exercise group 2.

sessions and in the focus on postural control (EG1). The lack of improvement in most of the FF parameters can be explained by the ceiling effect, mainly on the balance parameters. However, the motor skills developed in MAAP exercise intervention followed by EG2 led to an improved balance control, particularly by an increase in postural regulation as manifested in the observed decrease in plantar pressure. Moreover, the reduction of plantar pressure manifested in hindfoot means that the initial contact of the gait was done with greater movement control, which represents an important indicator of the impact on adverse outcomes, namely on the decrease of the risk of falling. On the other hand, both the elderly groups were quite fit at baseline, and exercise volume was not sufficient to promote a significant increase in overall FF in EG1, but only to improve fewer functional capacities and to maintain other in this group. The motor

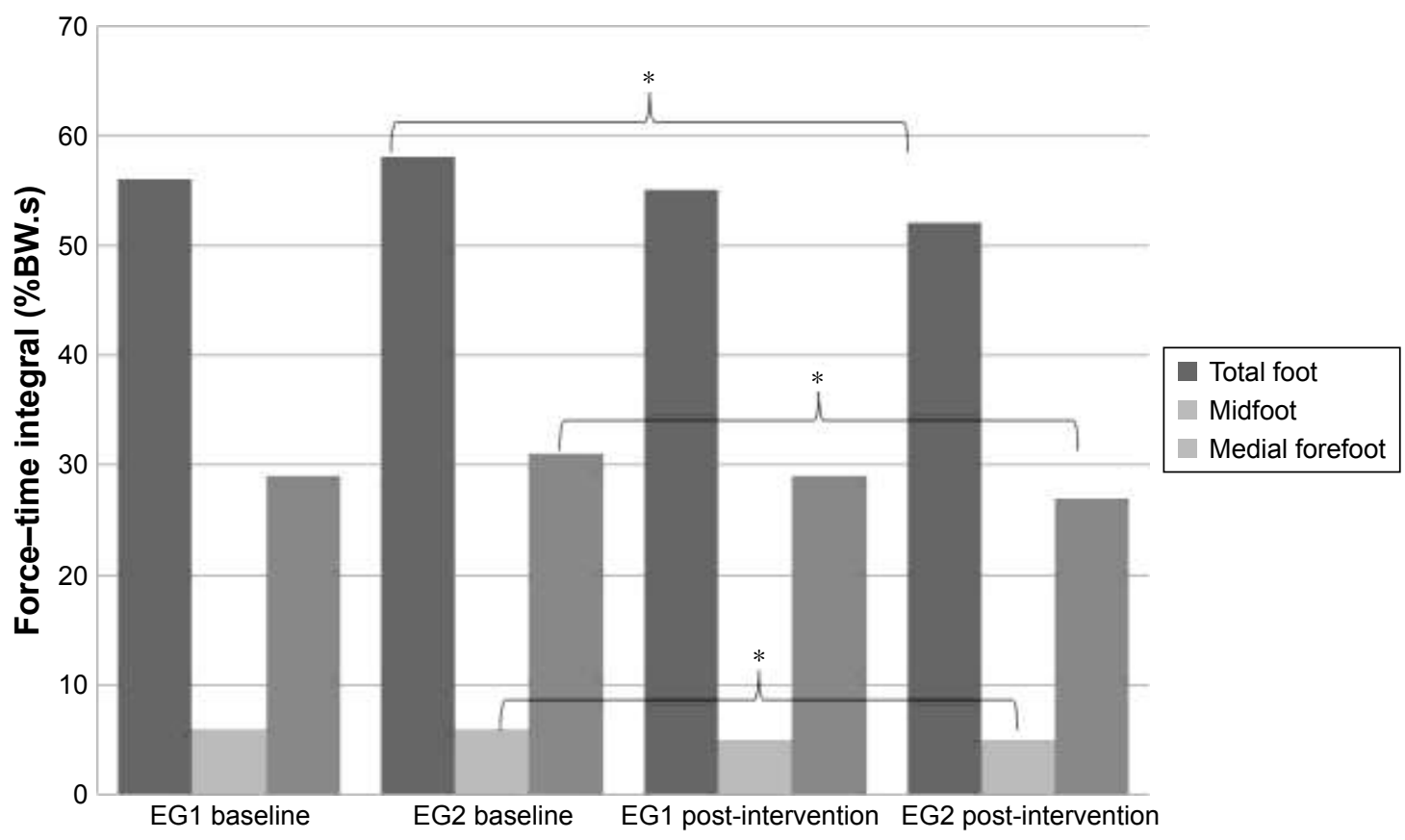

Figure 4 Significant changes between baseline and after intervention, in both the groups, regarding force-time integral $\left({ }^{*} p<0.05\right)$. Abbreviations: EGI, exercise group I; EG2, exercise group 2. 


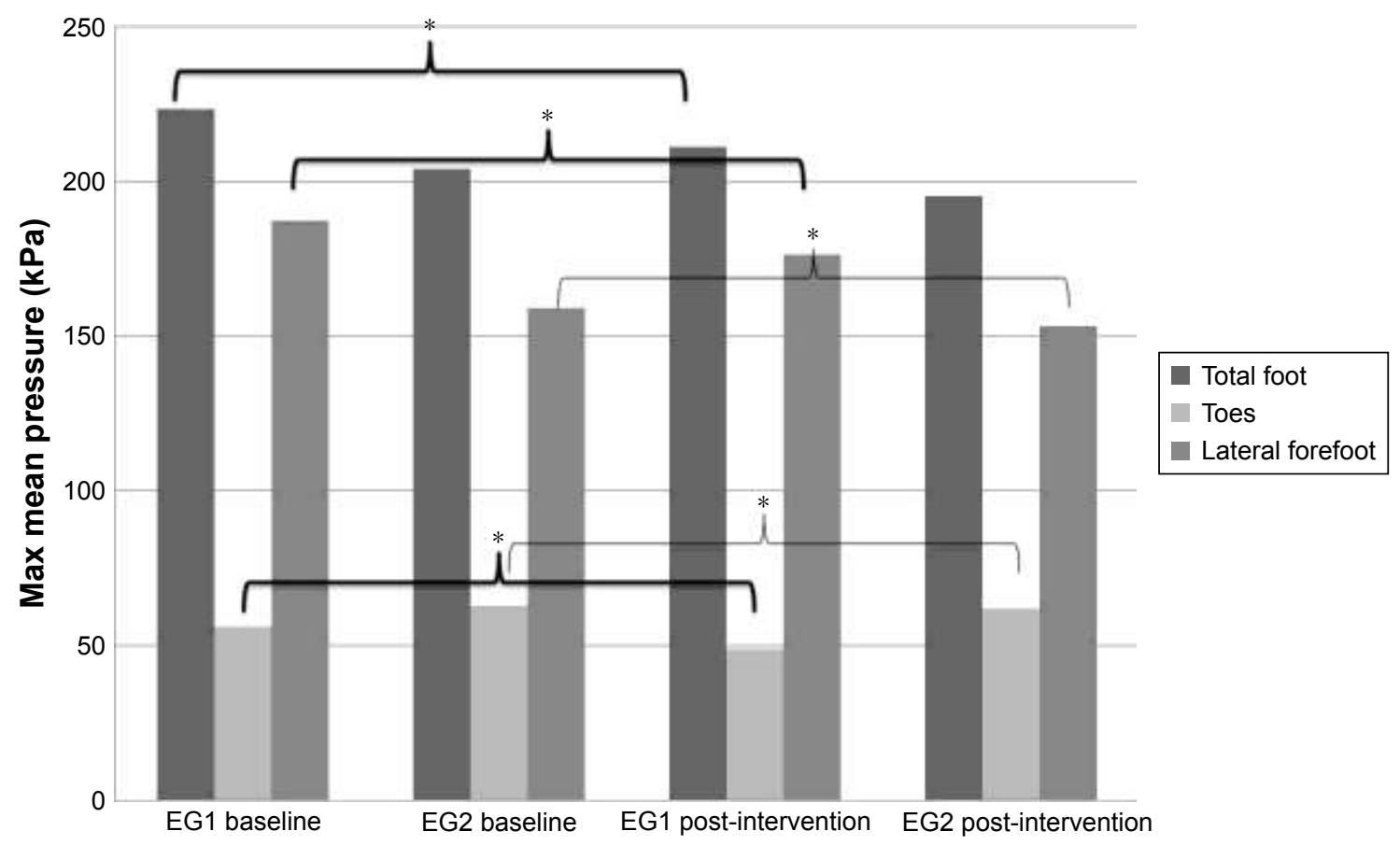

Figure 5 Significant changes between baseline and after intervention, in both the groups, regarding maximal mean pressure $\left({ }^{*} p<0.05\right)$. Abbreviations: EGI, exercise group I; EG2, exercise group 2.

skills developed in MAAP exercise intervention followed by EG2 led to an improved balance control, particularly by an increase in postural regulation as manifested in the observed decrease in plantar pressure. These results are quite similar to those obtained by Burton et al. ${ }^{29}$ In this line, the ACSM issued what is considered as the gold standard of exercise prescription for healthy old adults. ${ }^{23}$ However, a careful examination of this position stand suggests the number of

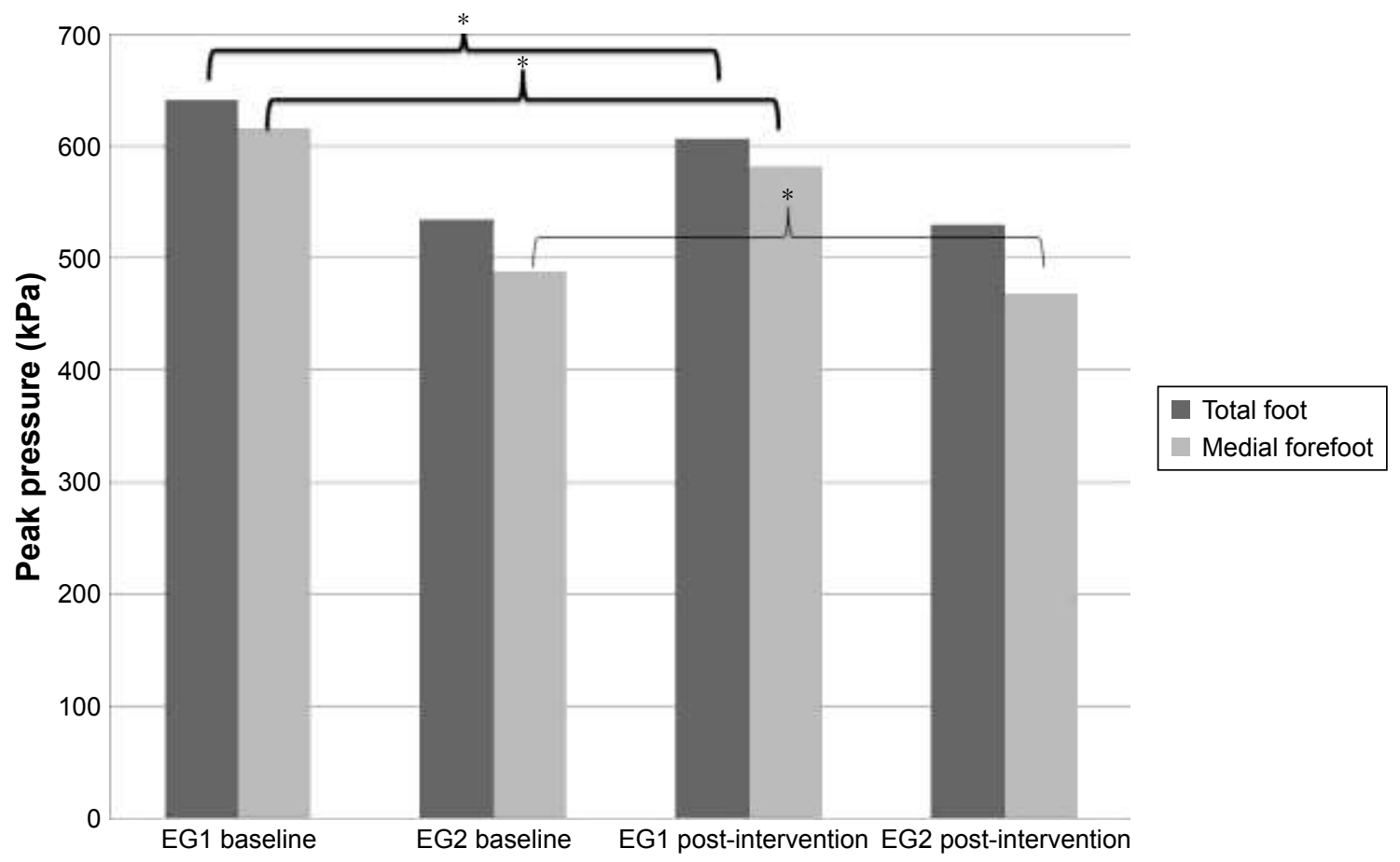

Figure 6 Significant changes between baseline and after intervention, in both the groups, regarding peak pressure $\left({ }^{*} p<0.05\right)$.

Abbreviations: EGI, exercise group I; EG2, exercise group 2. 


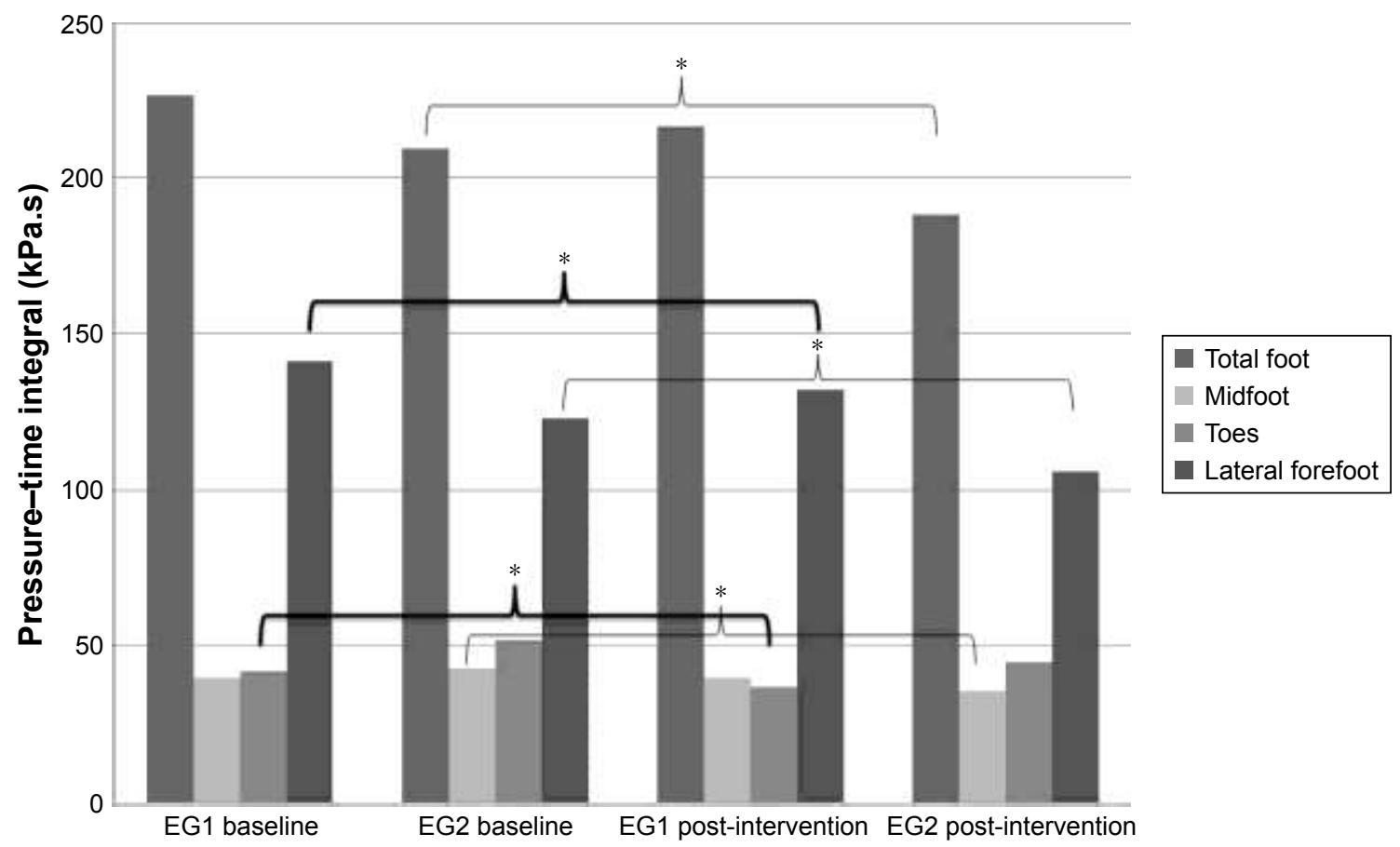

Figure 7 Significant changes between baseline and after intervention, in both the groups, regarding pressure-time integral $\left({ }^{*} p<0.05\right)$. Abbreviations: EGI, exercise group I; EG2, exercise group 2.

included training variables (eg, traditional variables such as training period, frequency, volume, intensity only), ${ }^{30}$ and by focusing only on direct comparisons of exercise groups (eg, high vs low intensity), it seems weak to measure the effectiveness of exercise programs. Moreover, the absence of reference values about the minimum detectable difference in the various dimensions of FF does not allow us to consider the conditions about the existence or not of improvements in the groups. In this study, this is especially important, because both the exercise groups included active older adults, and the quantification of the dose-response relationships/answers are more difficult to achieve.

More research is needed with studies using larger sample sizes, and longer follow-up periods, to establish evidencebased recommendations. However, our results regarding the main outcomes are important, considering that higher plantar pressure values have been associated with a change in walking strategy and an increase in fall risk. Gait impairment has frequently been detected in frail patients, especially a reduction in gait speed. ${ }^{3,31}$ However, there are other gait parameters such as the 10 spatiotemporal addressed by Ritt et al in a cross-sectional study. ${ }^{31}$ Moreover, those parameters might most accurately predict 1 -year mortality. ${ }^{32}$

We speculate that the long-term effectiveness of this type of exercise interventions will be established at the level of general mobility by means of adopting an active lifestyle, which can be translated into improved autonomy, independent living, and prevention of falls. ${ }^{33}$ The risk of falling associated with movement patterns in which elderly patients present higher GRF values in heel-contact (initial contact) may explain a higher plantar pressure in the hindfoot zone (which decreased in EG2). The results showed that the exercise programs, both periodized and non-periodized issues and tailored to older adults, were proved to be effective in reducing the age-related decline regarding FF and improve gait parameters. In this line, the efficacy of the exercise programs on the reduction of risk of falling in the long term is promising. . $^{14,33}$

To our knowledge, this is the first study investigating the effect of exercise and its organization (periodized vs nonperiodized program) on gait, based on peak forces and plantar pressures in independent community-dwelling older adults. Apart from this innovation, the major strength of this study was the ecological approach used, that is, the development and implementation of exercise interventions, combining the advantages of a community group program with the efficacy of controlled exercise and a progressive stimulus tailored to participant's FF level. The intervention was adapted to the typical community-based programs offered to the older population in rural areas and small towns. The programs were based on multiple component group exercise and showed great adherence, which in turn may contribute to compliance and long-term maintenance of older people.

Regarding the implications for practice, the present study has demonstrated that long-term periodized and 
non-periodized exercise programs tailored to older adults proved to be effective in reducing the age-related decline regarding FF and selected gait parameters. Both the exercise programs were safe, targeted to the physical fitness levels, and can be used by exercise professionals in prescribing community exercise programs, as well as by health professionals in promoting active aging and health among older adults. It is important to highlight the relevance of postural control exercises in exercise programs for elderly people, due to the positive repercussions that may have on the gait pattern, regardless the improvement of FF dimensions, leading to the prevention of falls in community-dwelling older adults.

Since an ecological approach was used, and as stated in the study protocol, the limitations of this study are related to the difficulties of randomization of the groups since older people were keener on participating in the program that provides a more convenient setting, either because it was not far from their homes or where were their friends. Moreover, the time-consuming events related to the assessment of different variables required extra time from the participants, and the loss of follow-up in this study was related to the second period of assessment. Future studies should have a larger sample size and a better randomized procedure to investigate the long-term effects of exercise training on gait loading parameters.

Further research should focus on increasing the number of participants and age intervals, allowing the training effects of MAAP program, between active and sedentary older adults, with differentiated functional capacity. The equipment and methodology used for gait analysis have progressed substantially in recent years. Thus, longitudinal studies are required to test which gait parameters, either kinetics or kinematics, are more sensitive to tailored exercise interventions.

\section{Conclusion}

Exercise programs tailored to older adults (periodized or non-periodized) are effective on mobility improvement and helps to maintain FF in elderly. An explanation for these effects in our study could be higher quality in the execution of exercises due to supervision. In fact, evaluation of the exercise diaries revealed similar mean stages of progression between groups. This implies that a higher rate of exertion and consequently a larger adaptation were achieved in both the experimental groups. The question remains as to why periodized program improved physical performance. It is possible that a higher dose needs to be applied in order to improve all functional parameters and thus a more effective improvement in gait.

\section{Acknowledgments}

The authors are grateful to all the seniors who voluntarily participated in this study.

This work was supported by:

- FCT - Portuguese Foundation for Science and Technology (project number PTDC/DES/72946/2006)

- European Union - QREN-INALENTEJO (reference number ALENT-07-0262-FEDER-001883)

The funding sources of the study had no role in the design, implementation, recruitment, data collection, analysis, or preparation of this manuscript.

\section{Author contributions}

All authors contributed toward data analysis, drafting and revising the paper and agree to be accountable for all aspects of the work.

\section{Disclosure}

The authors report no conflicts of interest in this work.

\section{References}

1. American College of Sports Medicine; Chodzko-Zajko WJ, Proctor DN, Fiatarone Singh MA, et al. American College of Sports Medicine position stand. Exercise and physical activity for older adults. Med Sci Sports Exerc. 2009;41(7):1510-1530.

2. Menz HB. Biomechanics of the ageing foot and ankle: a mini-review. Gerontology. 2015;61(4):381-388.

3. Schwenk M, Howe C, Saleh A, et al. Frailty and technology: a systematic review of gait analysis in those with frailty. Gerontology. 2014; 60(1):79-89.

4. Amemiya A, Noguchi H, Oe M, et al. Elevated plantar pressure in diabetic patients and its relationship with their gait features. Gait Posture. 2014;40(3):408-414.

5. O'Brien DL, Tyndyk M. Effect of arch type and body mass index on plantar pressure distribution during stance phase of gait. Acta Bioeng Biomech. 2014;16(2):131-135.

6. Mickle KJ, Munro BJ, Lord SR, Menz HB, Steele JR. Foot pain, plantar pressures, and falls in older people: a prospective study. J Am Geriatr Soc. 2010;58(10):1936-1940.

7. Fernando ME, Crowther RG, Lazzarini PA, et al. Plantar pressures are higher in cases with active diabetic foot ulcers compared to controls despite a longer stance phase duration. BMC Endocr Disord. 2016; 16(1):51.

8. Sullivan J, Burns J, Adams R, Pappas E, Crosbie J. Plantar heel pain and foot loading during normal walking. Gait Posture. 2015;41(2): 688-693.

9. Wang RY, Wang YL, Cheng FY, Chao YH, Chen CL, Yang YR. Effects of combined exercise on gait variability in community-dwelling older adults. Age (Dordr). 2015;37(3):9780.

10. Paterson DH, Warburton DE. Physical activity and functional limitations in older adults: a systematic review related to Canada's Physical Activity Guidelines. Int J Behav Nutr Phys Act. 2010;11(7):38.

11. van der Bij AK, Laurant MG, Wensing M. Effectiveness of physical activity interventions for older adults: a review. Am J Prev Med. 2002; 22(2):120-133.

12. Seco J, Abecia JC, Echevarría E, et al. A long-term physical activity training program increases strength and flexibility, and improves balance in older adults. Rehabil Nurs. 2013;38(1):37-47.

13. Wonneberger M, Schmidt S. Changes of gait parameters following long-term aerobic endurance exercise in mildly disabled MS patients: an exploratory study. Eur J Phys Rehabil Med. 2015;51(6):755-762. 
14. van Abbema R, de Greef M, Crajé C, Krijnen W, Hobbelen H, van der Schans C. What type, or combination of exercise can improve preferred gait speed in older adults? A meta-analysis. BMC Geriatrics. 2015; 15(1):72.

15. Chen MS, Lin TC, Jiang BC. Aerobic and resistance exercise training program intervention for enhancing gait function in elderly and chronically ill Taiwanese patients. Public Health. 2015;129(8): 1114-1124.

16. Chung MJ, Wang MJ. Gender and walking speed effects on plantar pressure distribution for adults aged 20-60 years. Ergonomics. 2012; 55(2):194-200.

17. Kim SG, Park JH. The effects of dual-task gait training on foot pressure in elderly women. J Phys Ther Sci. 2015;27(1):143-144.

18. Franco PS, Silva CB, Rocha ES, Carpes FP. Variability and repeatability analysis of plantar pressure during gait in older people. Rev Bras Reumatol. 2015;55(5):427-433.

19. Lalande X, Vie B, Weber JP, Jammes Y. Normal values of pressures and foot areas measured in the static condition. J Am Podiatr Med Assoc. 2016;106(4):265-272.

20. Monteiro MA, Gabriel RE, Neves E, et al. Exercise effects in plantar pressure of postmenopausal women. Menopause. 2010;17(5):1017-1025.

21. Strohacker K, Fazzino D, Breslin WL, Xu X. The use of periodization in exercise prescriptions for inactive adults: a systematic review. Prev Med Rep. 2015;2:385-396.

22. Ramalho F, Carnide F, Santos-Rocha R, et al. Community-based exercise intervention for gait and functional fitness improvement in an older population: study protocol. J Aging Phys Act. 2017;25(1):84-93.

23. American College of Sports Medicine. ACSM's Guidelines for Exercise Testing and Exercise Prescription. 10th ed. Philadelphia, PA: Wolters Kluwer/Lippincott, Williams and Wilkins; 2017.

24. Rose DJ. Fallproof!: A Comprehensive Balance and Mobility Training Program. 2nd ed. Champaign, IL: Human Kinetics; 2015.

25. Rikli RE, Jones CJ. Senior Fitness Test Manual. 2nd ed. Champaign, IL: Human Kinetics; 2013.
26. Moniz-Pereira V, Carnide F, Machado M, André HI, Veloso AP. Falls in Portuguese older people: procedures and preliminary results of the study Biomechanics of Locomotion in the Elderly. Acta Reumatol Port. 2012;37:324-332.

27. Valente $\mathrm{S}$. Validação de um questionário de saúde e identificação de factores de risco de quedas para a população idosa portuguesa [Validation of a health questionnaire and identification of fall risk factors among the Portuguese older population] [master's thesis]. Faculdade de Motricidade Humana - Universidade Técnica de Lisboa, CruzQuebrada; 2012.

28. Machado M, Tavares C,Moniz-Pereira V, AndréHI, RamalhoF, Veloso AP. Validation of YPAS-PT - The Yale Physical Activity Survey for Portuguese Older People. Sci J Public Health. 2016;4(1):72-80.

29. Burton E, Cavalheri V, Adams R, et al. Effectiveness of exercise programs to reduce falls in older people with dementia living in the community: a systematic review and meta-analysis. Clin Interv Aging. 2015; 10:421-434.

30. Raymond MJ, Bramley-Tzerefos RE, Jeffs KJ, Winter A, Holland AE. Systematic review of high-intensity progressive resistance strength training of the lower limb compared with other intensities of strength training in older adults. Arch Phys Med Rehabil. 2013;94(8): 1458-1472.

31. Ritt M, Schülein S, Lubrich H, Bollheimer LC, Sieber CC, Gassmann K-G. High-technology based gait assessment in frail people: associations between spatio-temporal and three-dimensional gait characteristics with frailty status across four different frailty measures. J Nutr Health Aging. 2017;21(3):346-353.

32. Ritt M, Ritt JI, Sieber CC, Gaßmann KG. Comparing the predictive accuracy of frailty, comorbidity, and disability for mortality: a 1-year follow-up in patients hospitalized in geriatric wards. Clin Interv Aging. 2017;12:293-304

33. Gillespie LD, Robertson MC, Gillespie WJ, et al. Interventions for preventing falls in older people living in the community. Cochrane Database Syst Rev. 2012;9:CD007146.
Clinical Interventions in Aging

\section{Publish your work in this journal}

Clinical Interventions in Aging is an international, peer-reviewed journal focusing on evidence-based reports on the value or lack thereof of treatments intended to prevent or delay the onset of maladaptive correlates of aging in human beings. This journal is indexed on PubMed Central, MedLine,
Dovepress

CAS, Scopus and the Elsevier Bibliographic databases. The manuscript management system is completely online and includes a very quick and fair peer-review system, which is all easy to use. Visit http://www.dovepress. com/testimonials.php to read real quotes from published authors. 